\title{
Transmission of Synovial Sarcoma from A Single Multiorgan Donor to Three Transplant Recipients: Cases Report and Literature Review
}

\section{Jian Zhang}

Capital Medical University: Beijing key laboratory of Tolerance Induction and Organ Protection in Transplantation

Ye Tian

Beijing Friendship Hospital, Capital Medical University

\section{Yang Yang}

Beijing Friendship Hospital, Capital Medical University

\section{Ruifang Xu}

Beijing Friendship Hospital, Capital Medical University

Jun Lin ( $\square$ bfhlinjun@hotmail.com )

Capital Medical University: Beijing key laboratory of Tolerance Induction and Organ Protection in Transplantation https://orcid.org/0000-0002-6847-7150

\section{Case report}

Keywords: Synovial sarcoma, Donor-derived malignancy, Donor transmission, Transplantation, Case report

Posted Date: November 23rd, 2020

DOI: https://doi.org/10.21203/rs.3.rs-77470/v1

License: (c) (1) This work is licensed under a Creative Commons Attribution 4.0 International License. Read Full License 


\section{Abstract}

Background Donor-derived malignancy is sometimes unidentified at the time of transplantation, resulting in unanticipated donor-derived malignancy, which becomes a notable problem since the increasing use of marginal donors.

Case presentation We first report three cases of donor-derived synovial sarcoma from a single multiorgan donor in China. The donor was died of respiratory failure caused by an intrathoracic tumor, which was diagnosed as a benign tumor at the time of donation. All of the three recipients developed synovial sarcoma within 3 months to 13 months after transplantation, proven to be donor-derived. Liver transplant recipient died of tumor metastasis. Two kidney transplant recipients survived from metastases by comprehensive therapy, including the first use of Anlotinib, exhibiting an effective anti-tumor activity.

Conclusions This report highlights the importance of detailed donor assessment, close follow-up and timely treatment, as well as the need for establishing a post-transplant surveillance system for donorderived transmission events. Whether to use organs from a donor with initial diagnosis of benign tumor needs to be cautious. Malignant potential of tumors needs to be excluded before transplantation, otherwise, the organs should be discarded.

\section{Background}

The persistent organ shortage requires the maximum utilization of all available donors, including those with tumors, that may lead to donor-derived malignancy ${ }^{1-3}$. There is a consensus that whether to use donor organs with tumors depends on risk levels of tumor transmission. However, a donor-derived malignancy is sometimes unidentified at the time of transplantation, resulting in unanticipated donorderived malignancy. Here, we first report three cases of donor-derived synovial sarcoma from a single multiorgan donor in China. In addition, risk of using organ donor with benign tumor is further discussed to help improving the safety of transplantation.

\section{Case Presentation}

\section{Transplant recipients}

In 2018, three recipients from a single multiorgan donor developed allograft synovial sarcoma within 3 months to 13 months consecutively after organ transplantation. Timeline was displayed in Fig. 1.

Synovial sarcoma was initially developed in allograft liver of recipient 1 three months after transplantation, who was a 26-year-old female with primary liver cancer. However, the donor-derived transmission was not recognized. The patient received a resection of left lateral lobe of allograft liver. Recurrence in her right lobe of allograft liver was found 11 months after partial allograft hepatectomy. 3 months later, systemic metastases were found and she died within 2 months. 
Recipient 2 was a 43-year-old male who received the left kidney. 9 months after transplantation, multiple neoplasms in the allograft kidney were found by ultrasound and computed tomography (CT) scan. Positron emission tomography with computed tomography (PET/CT) revealed a tendency to malignancy, without metastasis. Synovial sarcoma was identified after biopsy and the patient received allograft nephrectomy, followed by withdrawal of immunosuppression. The cancer was finally proven to be donorderived by DNA microsatellite. 4 months after nephrectomy, CT examination revealed diffuse pulmonary metastases. He received targeted therapy with Anlotinib, which exhibited an effective anti-tumor activity with the elimination of metastases. The patient was alive without cancer progression during two-year follow-up.

With the warning of transmission from the same donor, a regular cancer screening was performed for recipient 3 who was a 33-year-old male and received the right kidney. Unfortunately, he developed a single neoplasm in the allograft kidney 3 months after the warning. Biopsy pathology revealed the same result with recipient 2 . He initially received radiofrequency ablation in order to preserve the allograft function. However, local recurrence was found 6 months later. The patient received allograft nephrectomy, followed by withdrawal of immunosuppression. Donor-derived malignancy was proven by DNA microsatellite. Half a month later, CT examination revealed diffuse pulmonary metastases and he received targeted therapy with Anlotinib, which again exhibited an excellent efficacy. The patient was alive without cancer progression during almost two-year follow-up.

\section{Organ donor}

The donor was a 14-year-old girl who developed a $11 \mathrm{~cm}$ intrathoracic tumor. In October 2017, she died of respiratory failure caused by tracheal compression and donated a liver and two kidneys. Hematoxylin and eosin (HE) staining by biopsy before organ procurement revealed a benign tumor. Immunohistochemical staining revealed solitary fibrous tumor.

\section{Imageological finding}

Multiple solid hypo-echo neoplasms were found with punctiform blood flow signal inside the allograft kidney in recipient 2 (Fig. 2A), as well as in recipient 3 (Fig. 2E), by color Doppler ultrasound. Compared with the density of allograft, a slightly higher density of neoplasm was found in non-enhancing $\mathrm{CT}$, but a slow and relatively homogeneous enhancement with lower density inside the neoplasm was found in enhanced multiphase images in both recipient 2 (Fig. 2B-D) and recipient 3 (Fig. 2F-H). There was no evidence of metastases on CT examination.

\section{Pathological finding}

Histopathology revealed monotonous proliferation of spindle-shaped cells on HE staining. On immunohistochemical staining, these cells were positively stained with TLE1, Vimentin, Bcl-2 and CD99, but were negative for CK, CD34, INI1, Desmin, MyoD1, WT-1 and S-100 protein. Molecular analysis confirmed the presence of SS18-SSX fusion, using FISH test (Fig. 3). 


\section{DNA microsatellite}

The length of detection locus in different tissues was shown in Fig. 4. Amel loci exhibited a female gender in both allograft and cancer tissues, confirming the donor-derived transmission.

\section{Discussion And Conclusion}

Transplantation from a donor with malignancy could produce a valuable increase in organs with a reasonable value of safety ${ }^{4,5}$. The reported risk of transmission varied from 0.01 to $0.05 \%{ }^{4-6}$. Despite of a low risk, it has been intermittently reported, resulting in fatal consequences $1-3,7,8$. This was the first reported synovial sarcoma transmission through a multiorgan procedure from 1 donor to 3 recipients.

The identification of synovial sarcoma remained a challenge due to histological overlap with other tumor types, mainly based upon a combination of traditional morphology, identification of the chromosomal $t(X ; 18)$ translocation and a panel of immunohistochemical markers ${ }^{9}$. Due to the limitation of diagnostic capacity or emergency of donation, misdiagnosis of malignancy before organ procurement might directly result in the use of malignant donor organs. In this study, synovial sarcoma was unidentified at the time of donation, leading to the subsequent unanticipated donor-derived transmission, that indicated the importance of systemic donor assessment. Due to the unrecognizability of some uncommon tumors or malignant potential, whether to use organs from a donor with initial diagnosis of benign tumor needs to be cautious. A list of benign tumors with malignant potential or other factors related to organ transplantation was summarized in Table 1. Donors with these tumors were recommended to receive a more detailed assessment to exclude malignant potential, otherwise, these donors should be discarded.

It remained speculative regarding the donor-derived transmission of malignancy from the donor without any signs of metastasis. Micro-metastasis of malignancy was considered as one hypothesis. Previous study demonstrated a greater risk for metastases of larger synovial sarcoma ${ }^{10}$. Given the tumor size of donor in this study, micro-metastasis was considered to be existing before organ procurement procedure. Another hypothesis was that changes of immune system in cancer surveillance might promote development of malignancy, due to the use of immunosuppressant ${ }^{11-13}$. On this account, the synovial sarcoma grew faster after organ transplantation.

In this study, all recipients eventually developed synovial sarcoma, revealing a high transmission rate. Previous study suggested that timely removal of allograft might be beneficial to prevent the development of metastasis ${ }^{2}$. In order to prevent transmission, it should be considered in all recipients after notification of cancer transmission in one recipient from a multiorgan donor. In this study, if the donor-derived transmission was recognized and warned earlier, we might prevent the transmission events by earlier removing the allograft.

Synovial sarcoma was considered an aggressive sarcoma, noted for its propensity of local recurrence and metastasis, with a poor prognosis owing to its resistance to radiation and chemotherapy. The 
treatment of allograft synovial sarcoma was rarely reported. However, several therapeutic options for allograft renal mass were listed, including partial nephrectomy, transplant nephrectomy, radiofrequency ablation and cryoablation, followed by altered or withdraw of immunosuppression ${ }^{14}$. Numbers of transplant surgeons and urologists faced with dilemma that required maximizing preservation of renal function while ensuring adequate cancer control. This study suggested that compared with local therapy, allograft nephrectomy followed by withdrawal of immunosuppression might be the best therapeutic option and more beneficial to local recurrence, which was supported by previous study ${ }^{3}$. Nevertheless, it did not prevent the development of metastasis, probably owing to the above-mentioned delayed treatment. Anlotinib was a new oral tyrosine kinase inhibitor, designed to primarily inhibit multi-targets in vasculogenesis and angiogenesis, which exhibited direct anti-tumor activity towards synovial sarcoma ${ }^{15}$. In this study, Anlotinib was proved to be effective towards synovial sarcoma by the elimination of metastases and prolonged progression-free survival of two kidney transplant recipients. Further observation of efficacy, drug resistance and safety were needed.

Donor-derived transmission of malignancy is not merely case report, but a notable public problem, which is considered to be underestimated, deserving much attention. Since 2005, Organ Procurement and Transplantation Network (OPTN) policy has required reporting of all unanticipated potential donor-derived transmission events in support of efforts to track, understand and learn from donor-derived disease transmission events in the United States ${ }^{16}$. China has made efforts to established several official transplant registry systems for post-transplant surveillance, nonetheless separated from each other that lack of systemic surveillance for donor-derived transmission events. Given the importance of timely notification to all risky recipients, it is necessary and urgency to establish a systemic surveillance system specially for donor-derived cancer transmission events in the current era of deceased donor organ transplantation.

This report highlights the importance of detailed donor assessment for preventing donor-derived malignancy, as well as the need for establishing a post-transplant surveillance system specially for donor-derived transmission events in the current era of deceased donor organ transplantation. Whether to use organs from a donor with initial diagnosis of benign tumor needs to be cautious. Malignant potential of tumors needs to be excluded before transplantation, otherwise, the organs should be discarded. Timely removal of allograft is the best option to prevent and cure donor-derived cancer transmission that should be considered in all recipients after notification of transmission in one recipient from a multiorgan donor. Besides, Anlotinib is proved to be effective towards synovial sarcoma.

\section{Abbreviations}

$\mathrm{CT}$, computed tomography

PET/CT, positron emission tomography with computed tomography

$\mathrm{HE}$, hematoxylin and eosin 
FISH, fluorescence in situ hybridization

PCR, polymerase chain reaction

OPTN, Organ Procurement and Transplantation Network

\section{Declarations}

\section{Ethical approval and consent to participate}

This study was conducted according to the ethical guidelines of the Helsinki Declaration and approved by the Ethics Committee of Beijing Friendship Hospital, Capital Medical University. Informed consent was waived that the information was anonymized and the submission did not include images that might identify the person.

\section{Consent for publication}

Consent for publication was waived that the information was anonymized and the submission did not include images that might identify the person.

\section{Competing interests}

The authors declare that they have no conflict of interest.

\section{Funding}

This study was funded by Beijing Natural Science Foundation (No. 7192043).

\section{Authors' contributions}

ZJ: Designed research, analyzed data and wrote paper

TY: Revised paper

YY: Collected data and analyzed data

XR: Collected data

LJ: Designed research, performed research and revised paper

\section{Acknowledgments}

We are grateful to Beijing Friendship Hospital, Capital Medical University for providing data for this study.

\section{Availability of data and materials}

All clinical data generated during this study are included in this article. 


\section{References}

1. Green M, Covington S, Taranto S, et al. Donor-derived transmission events in 2013: a report of the Organ Procurement Transplant Network Ad Hoc Disease Transmission Advisory Committee. Transplantation. 2015;99(2):282-7.

2. Matser YAH, Terpstra ML, Nadalin S, et al. Transmission of breast cancer by a single multiorgan donor to 4 transplant recipients. Am J Transplant. 2018;18(7):1810-4.

3. Xiao D, Craig JC, Chapman JR, et al. Donor cancer transmission in kidney transplantation: a systematic review. Am J Transplant. 2013;13(10):2645-52.

4. Desai R, Collett $D$, Watson $C J$, et al. Cancer transmission from organ donors-unavoidable but low risk. Transplantation. 2012;94(12):1200-7.

5. Eccher A, Lombardini L, Girolami I, et al. How safe are organs from deceased donors with neoplasia? The results of the Italian Transplantation Network. J Nephrol. 2019;32(2):323-30.

6. Myron Kauffman H, McBride MA, Cherikh WS, et al. Transplant tumor registry: donor related malignancies. Transplantation. 2002;74(3):358-62.

7. Baudoux TER, Gastaldello K, Rorive S, et al. Donor Cancer Transmission in Kidney Transplantation. Kidney Int Rep. 2016;2(2):134-7.

8. Lipshutz GS, Mihara N, Wong R, et al. Death from metastatic donor-derived ovarian cancer in a male kidney transplant recipient. Am J Transplant. 2009;9(2):428-32.

9. El Beaino M, Araujo DM, Lazar AJ, et al. Synovial Sarcoma: Advances in Diagnosis and Treatment Identification of New Biologic Targets to Improve Multimodal Therapy. Ann Surg Oncol. 2017;24(8):2145-54.

10. Przybyl J, Sciot R, Wozniak A, et al. Metastatic potential is determined early in synovial sarcoma development and reflected by tumor molecular features. Int J Biochem Cell Biol. 2014;53:505-13.

11. Koff JL, Waller EK. Improving cancer-specific outcomes in solid organ transplant recipients: Where to begin? Cancer. 2019;125(6):838-42.

12. Schreiber RD, Old LJ, Smyth MJ. Cancer immunoediting: integrating immunity's roles in cancer suppression and promotion. Science. 2011;331(6024):1565-70.

13. Crespo E, Fernandez L, Lúcia M, et al. Effector Antitumor and Regulatory T Cell Responses Influence the Development of Nonmelanoma Skin Cancer in Kidney Transplant Patients. Transplantation. 2017;101(9):2102-10.

14. Griffith JJ, Amin KA, Waingankar N, et al. Solid Renal Masses in Transplanted Allograft Kidneys: A Closer Look at the Epidemiology and Management. Am J Transplant. 2017;17(11):2775-81.

15. Tang L, Yu W, Wang Y, et al. Anlotinib inhibits synovial sarcoma by targeting GINS1: a novel downstream target oncogene in progression of synovial sarcoma. Clin Transl Oncol. 2019;21(12):1624-33.

16. Ison MG, Hager J, Blumberg E, et al. Donor-derived disease transmission events in the United States: data reviewed by the OPTN/UNOS Disease Transmission Advisory Committee. Am J Transplant. 
2009;9(8):1929-35.

\section{Tables}


Table 1

Benign tumors with malignant potential or other factors related to organ transplantation

Histological type Site Comment

\begin{tabular}{lll}
$\begin{array}{l}\text { Sclerosing } \\
\text { pneumocytoma }\end{array}$ & Lung & $\begin{array}{l}\text { Potential lymph node metastasis, distant metastasis or } \\
\text { malignant transformation. }\end{array}$ \\
$\begin{array}{l}\text { Perivascular } \\
\text { epithelioid cell } \\
\text { tumor }\end{array}$ & $\begin{array}{l}\text { Lung, } \\
\text { Gastrointestinal } \\
\text { tract, Uterine, } \\
\text { Sinonasal }\end{array}$ & $\begin{array}{l}\text { Difficult to distinct from malignant neoplasm. Tumor size }>5 \\
\text { cm, infiltrative growth pattern, high nuclear grade, necrosis, } \\
\text { and mitotic activity }>1 / 50 \text { HPF were identified for predicting } \\
\text { aggressive clinical behavior. }\end{array}$ \\
\hline $\begin{array}{l}\text { Epithelioid } \\
\text { angiomyolipoma }\end{array}$ & Kidney & $\begin{array}{l}\text { Reported malignant potential and distant metastasis. } \\
\text { Younger age, male sex, and larger tumor mass might increase } \\
\text { the possibility of diagnosis contribute to the poor prognosis. }\end{array}$ \\
$\begin{array}{l}\text { Pleomorphic } \\
\text { adenoma }\end{array}$ & Salivary gland & $\begin{array}{l}\text { Rare cases of histologically benign appearing pleomorphic } \\
\text { adenoma demonstrated metastatic behavior, that were called } \\
\text { benign metastasizing pleomorphic adenoma. Potential } \\
\text { malignant transformation and distant metastasis. }\end{array}$
\end{tabular}

$\begin{array}{ll}\text { Cystadenoma } & \text { Salivary gland, } \\ \text { Biliary tract }\end{array}$

A tumor with a benign histological appearance but could present with metastasis and behave in a clinically malignant manner. Difficult to distinct from cystadenocarcinoma and mucoepidermoid carcinomas.

Meningioma Intraspinal,

Potential malignant transformation and distant metastasis. Intracranial

Risk factors included high-dose radiation, surgical stress, viral infection, growth factors such as VEGF, and numerous progression-associated alternations of chromosomes $1 p, 6 q$, $9 q, 10 q, 14 q, 17 q$, and $22 q$.

Paraganglioma Orbit, Rectum

Potential malignant transformation and metastasis. However, there were no reliably predictable histologic criteria.

$\begin{array}{ll}\text { Gangliocytic } & \text { Pancreas, } \\ \text { paraganglioma } & \text { Duodenum }\end{array}$

Reported malignant potential and distant metastasis. Compared with duodenal GPs, pancreatic GPs were larger and had a higher incidence of metastasis, suggesting a greater potential for malignancy.

$\begin{array}{lll}\text { Solitary fibrous } & \text { Thyroid gland, } & \text { Potential malignant transformation and possibility of } \\ \text { tumor } & \text { Central nervous } & \text { metastasis. Several risk stratification models based on } \\ \text { system, Liver, } & \text { combining factors such as patient age, tumor size, mitotic } \\ & \text { Orbit, Thoracic } & \text { count, cellularity, pleomorphism, tumor necrosis, and tumor } \\ \text { cavity, } & \text { site had been proposed as a means of predicting clinical } \\ \text { Pancreas } & \text { outcome. }\end{array}$

Macrocystic Pancreas

serous

cystadenomas

Reported malignant potential and distant metastasis. Difficult to distinguish from benign counterparts by histological feature. Several parameters had been investigated to identify any predictive factor for aggressive behavior, such as tumor diameter $>6 \mathrm{~cm}$ and tumor location in the head of the pancreas.

Oncocytoma Kidney

Potential transformation to a high-grade oncocytic carcinoma and metastasis.

Adenoma Liver, Pituitary

Potential malignant transformation and metastasis. 


\section{Histological type Site Comment}

Schwannoma Vestibulum

Potential malignant transformation and difficult to determine the risk.

Figures

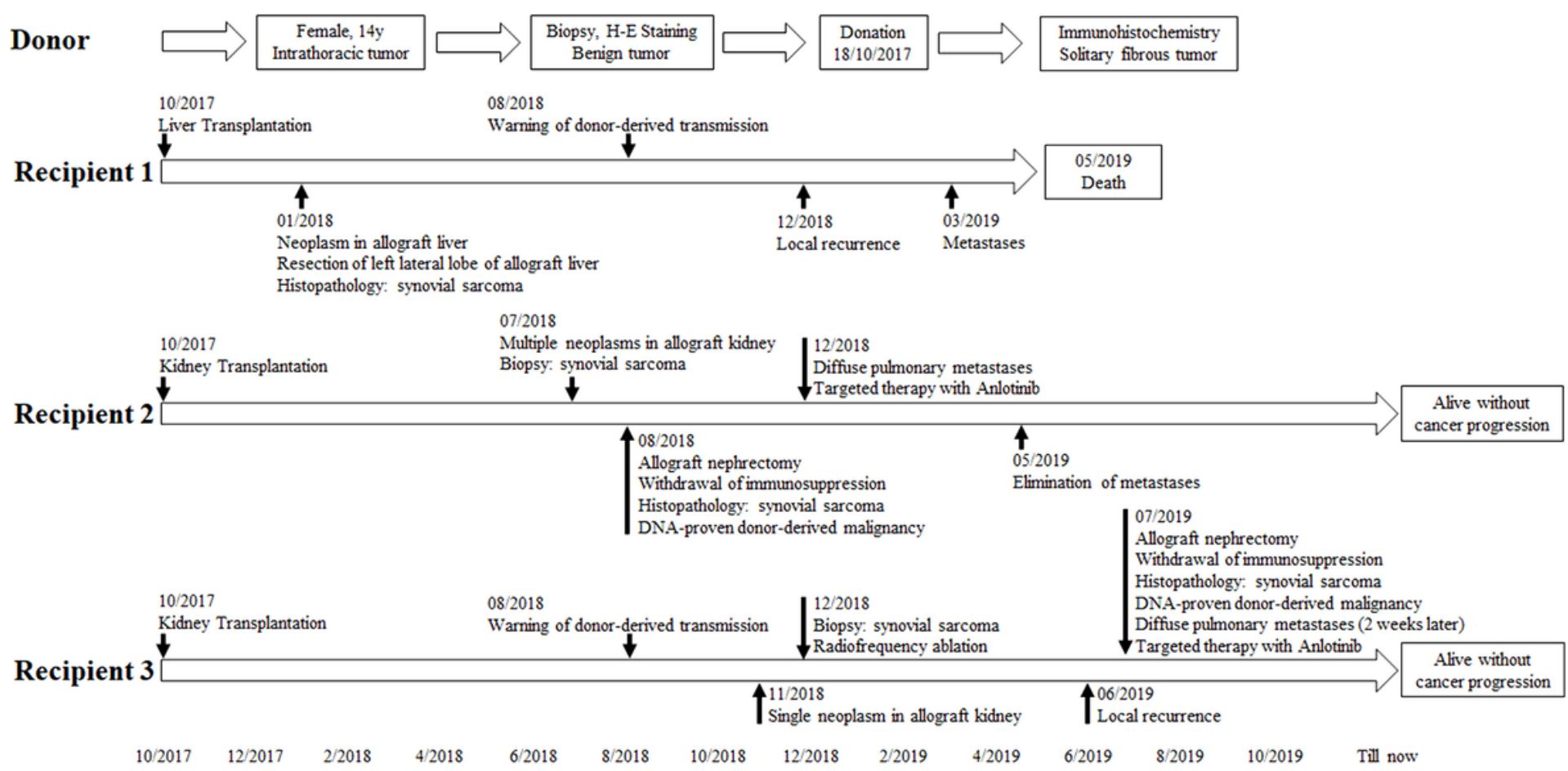

Figure 1

Clinical course of three recipients with donor-derived synovial sarcoma 


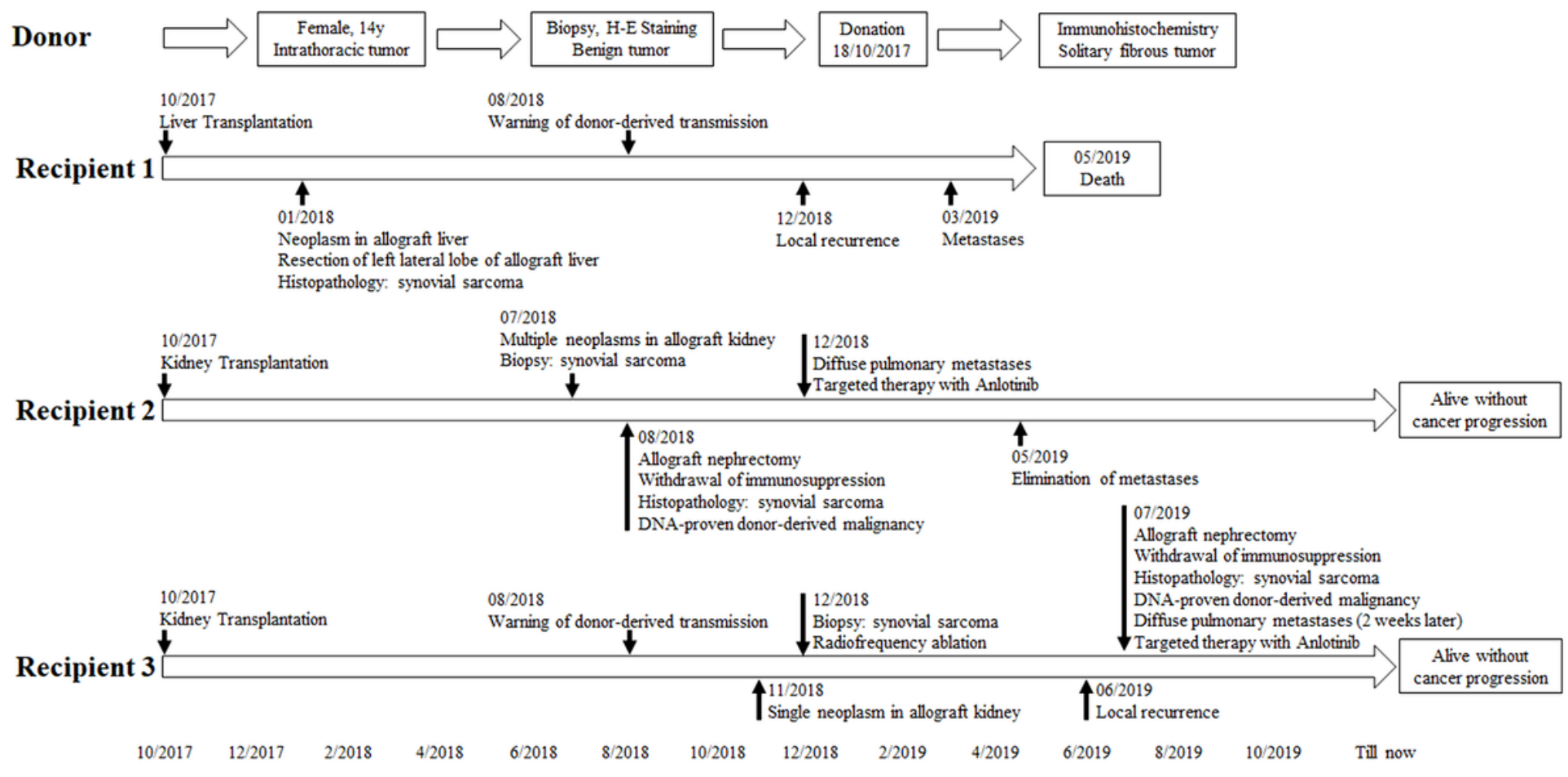

Figure 1

Clinical course of three recipients with donor-derived synovial sarcoma 

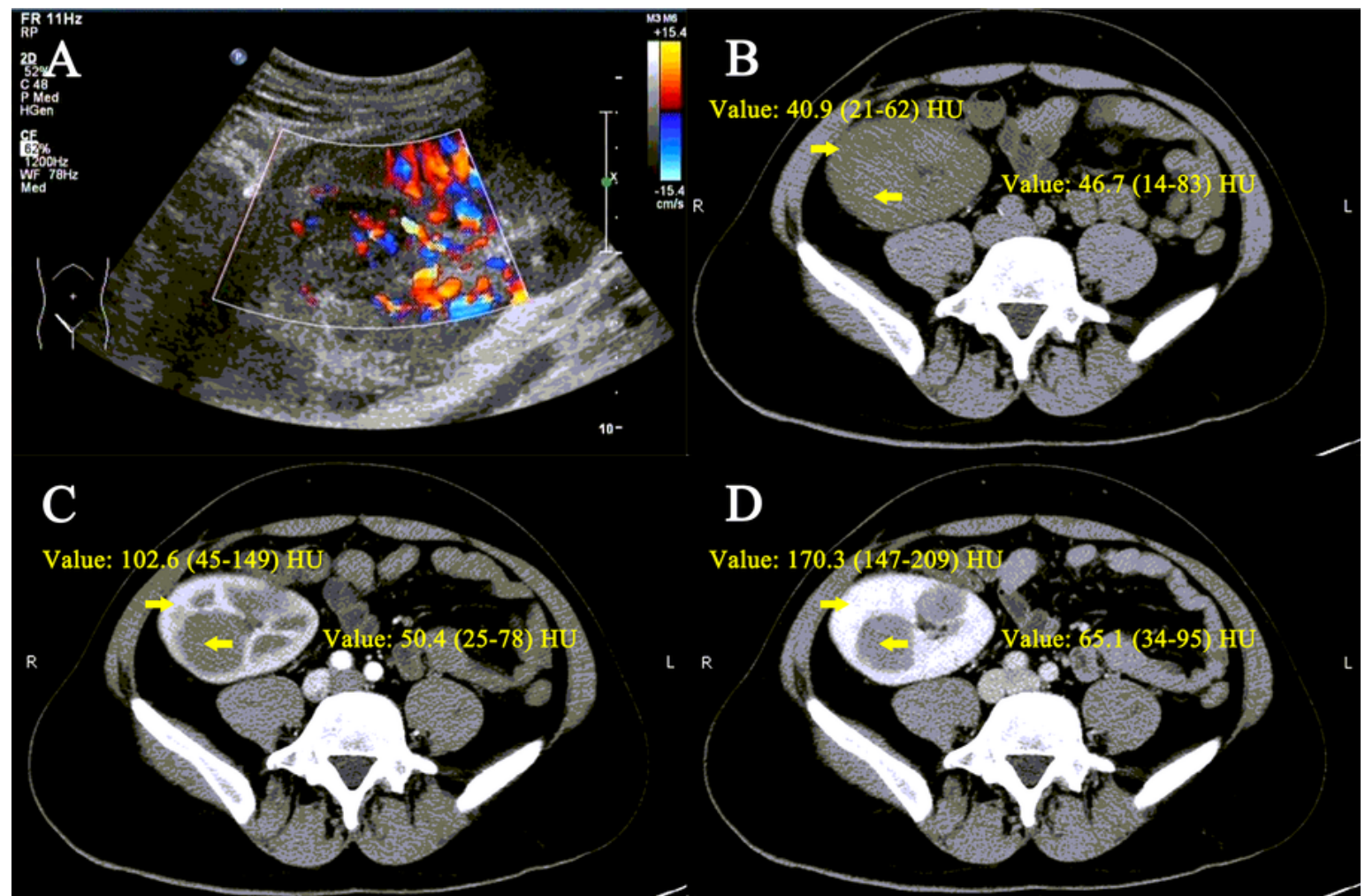

\section{D}
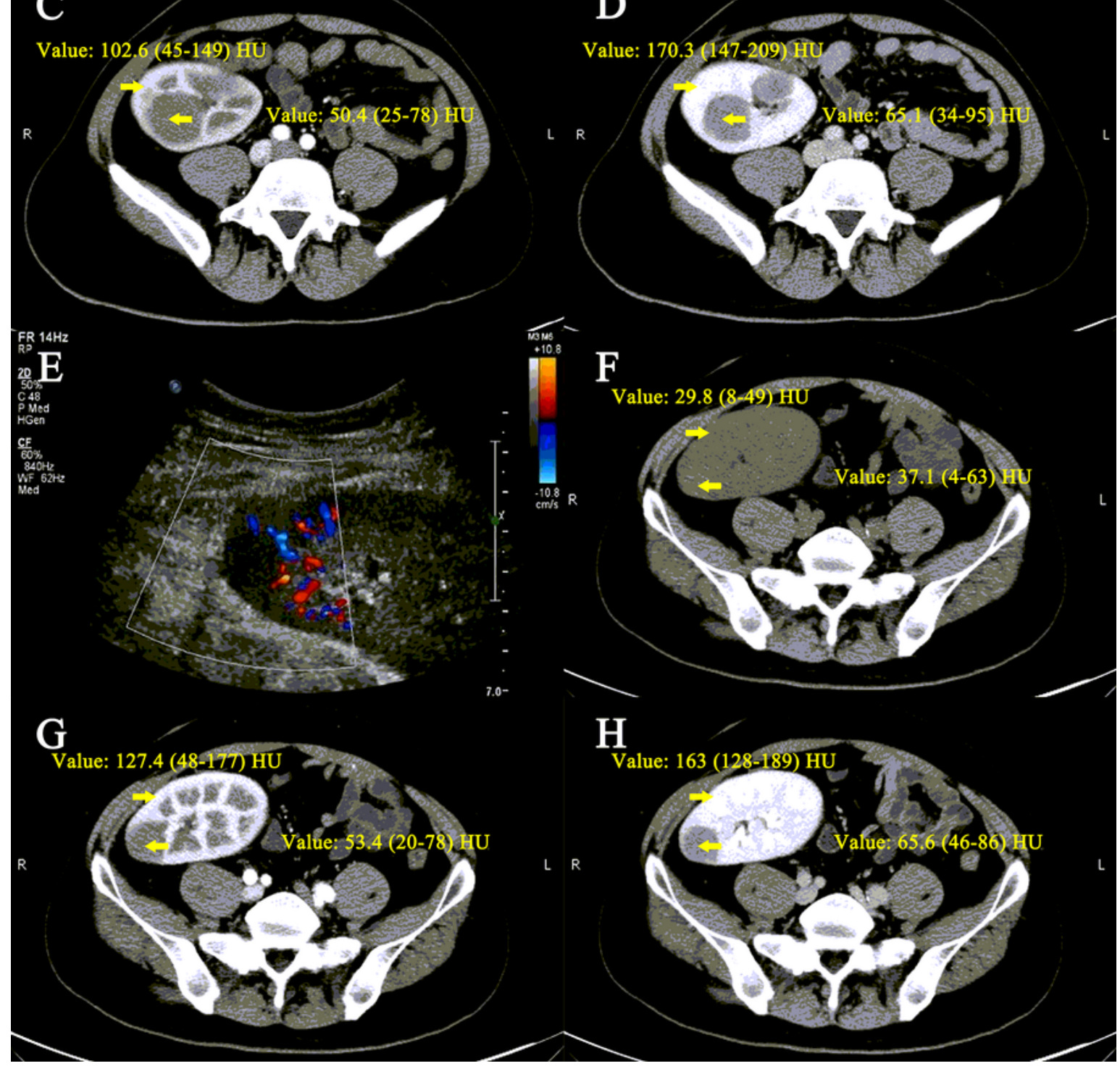

H

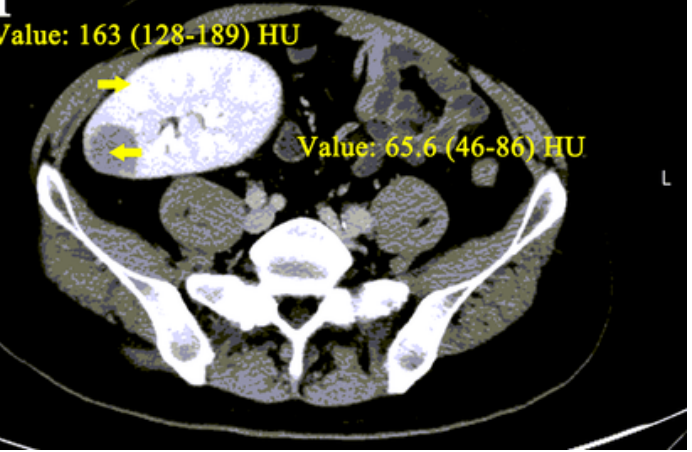

\section{Figure 2}

Ultrasonography and CT manifestation of allograft synovial sarcoma A and E: Color Doppler flow images revealed solid hypo-echo neoplasm with punctiform blood flow signal inside; B and F: Non-enhancing CT revealed slightly higher density of neoplasm than the allograft; C, D, G, H: Enhanced multiphase images revealed slow and relatively homogeneous enhancement inside the neoplasm, with lower density than the allograft. 

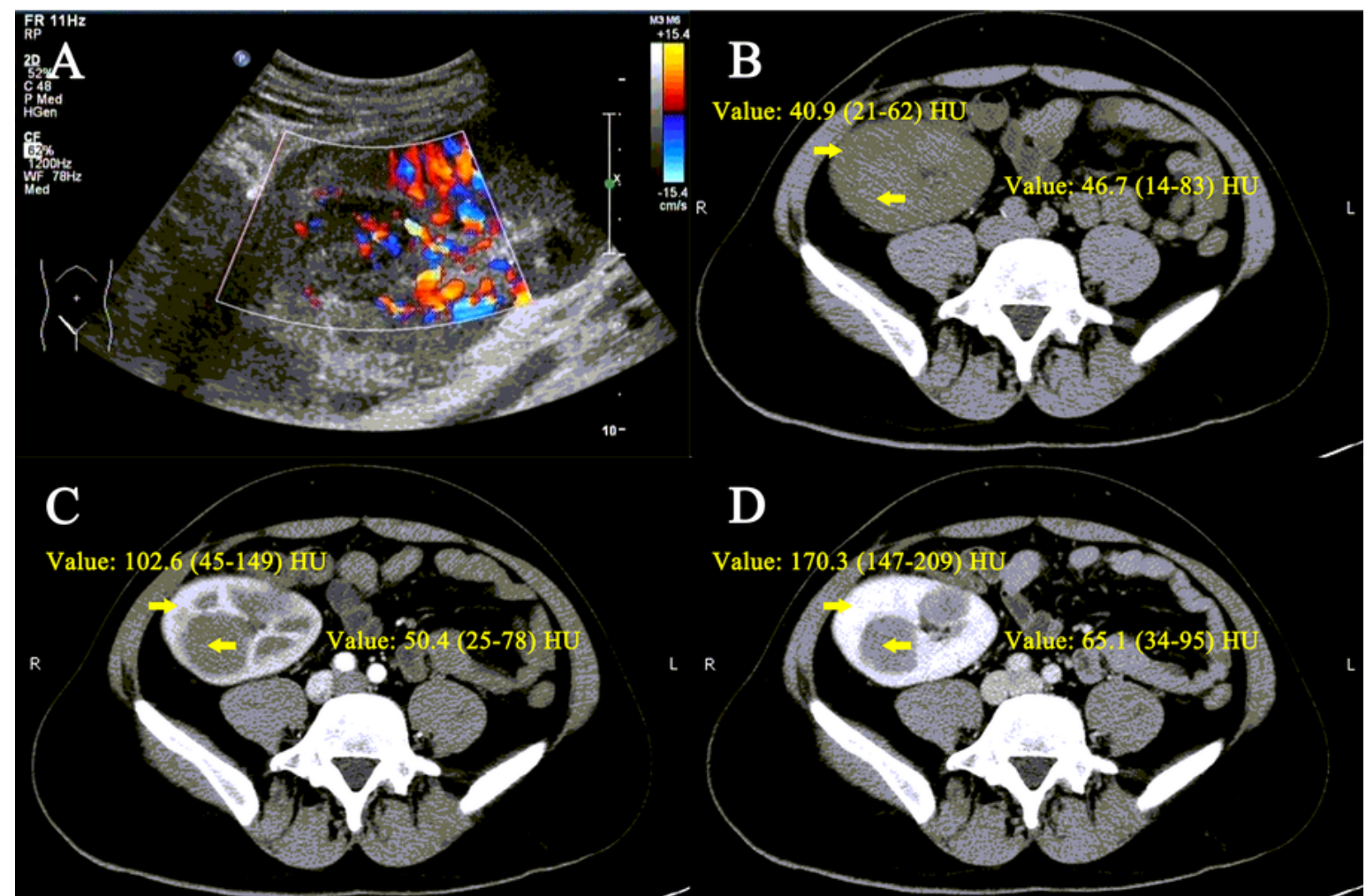

\section{D}
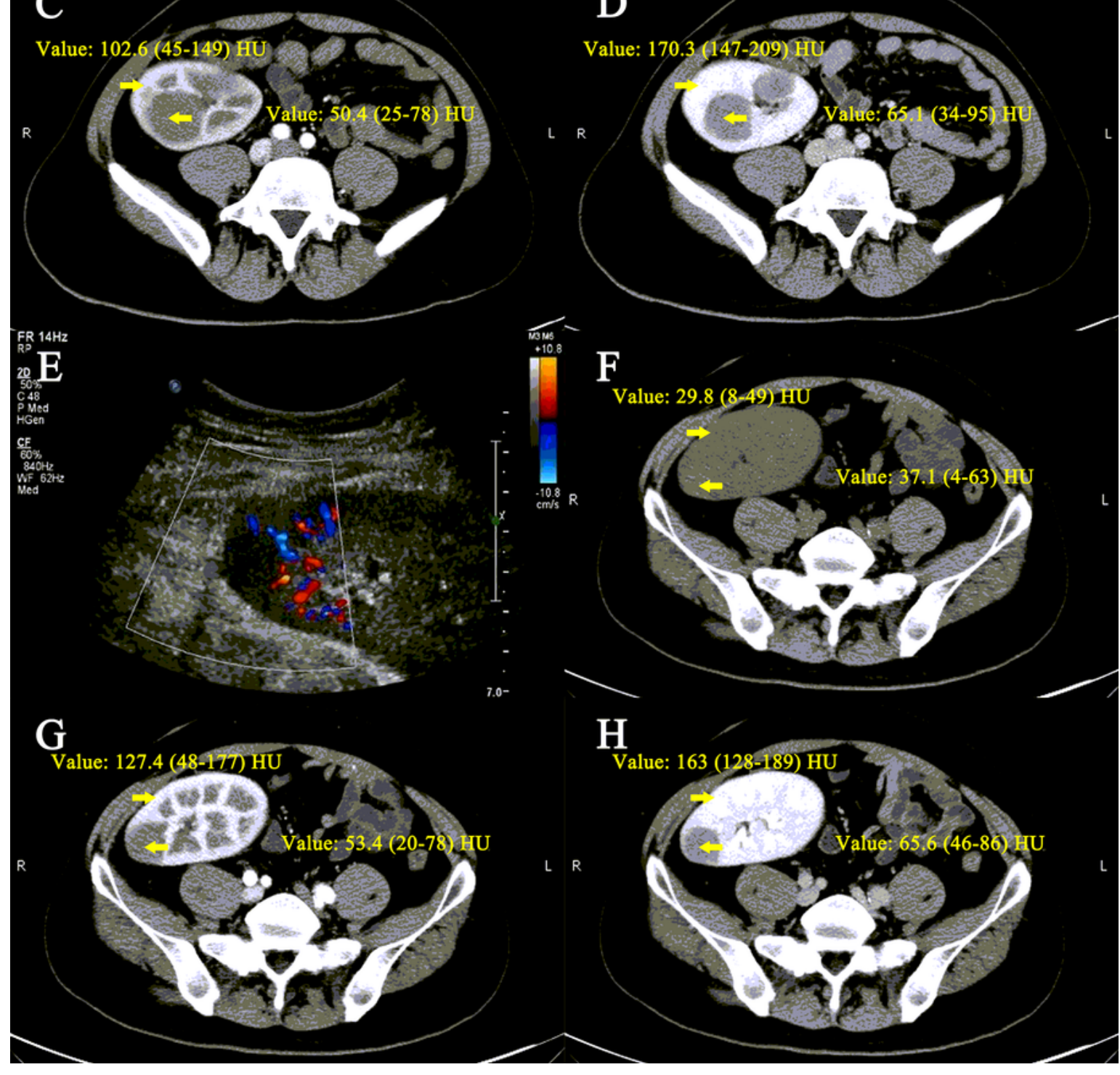

H

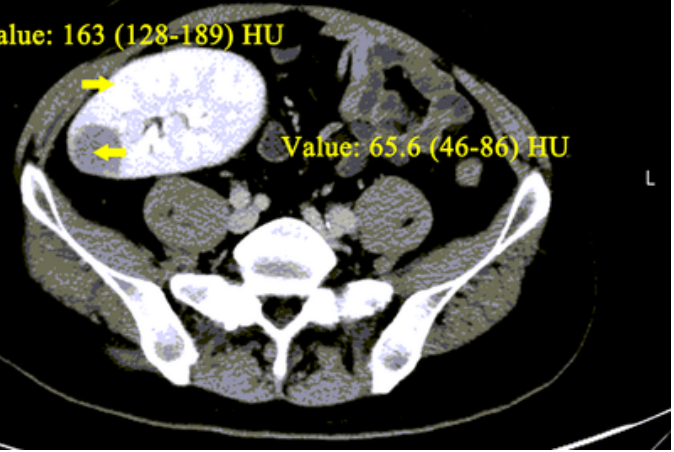

\section{Figure 2}

Ultrasonography and CT manifestation of allograft synovial sarcoma A and E: Color Doppler flow images revealed solid hypo-echo neoplasm with punctiform blood flow signal inside; B and F: Non-enhancing CT revealed slightly higher density of neoplasm than the allograft; C, D, G, H: Enhanced multiphase images revealed slow and relatively homogeneous enhancement inside the neoplasm, with lower density than the allograft. 

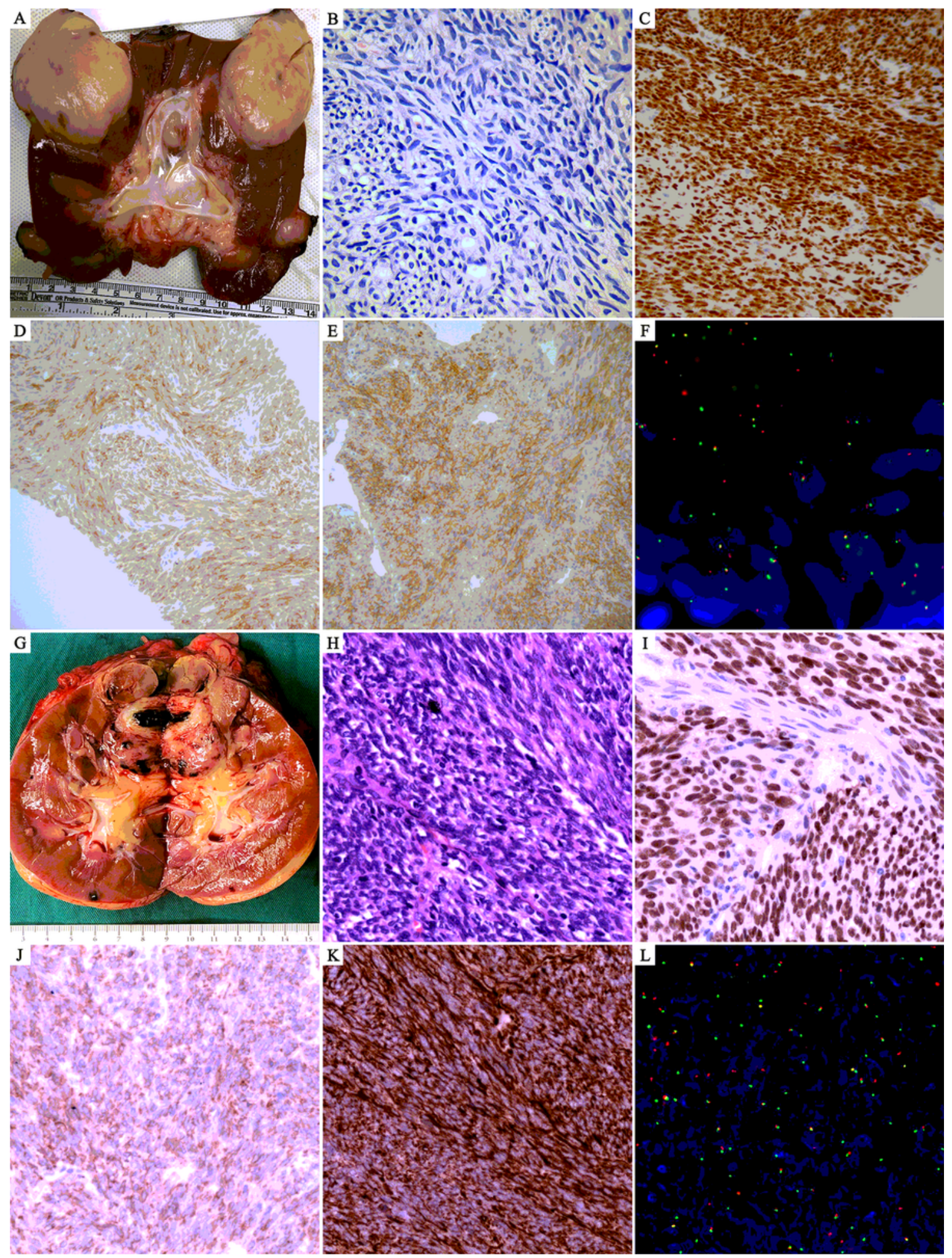

Figure 3

Histopathology of allograft synovial sarcoma Recipient 2: A: Specimen; B: HE-staining (200x); C: TLE1 (100x); D: Bcl-2 (100x); E: CD99 (200x); F: FISH test; Recipient 3: G: Specimen; H: HE-staining (200x); I: TLE1 (200x); J: Bcl-2 (200x); K: Vimentin (200x); L: FISH test. 

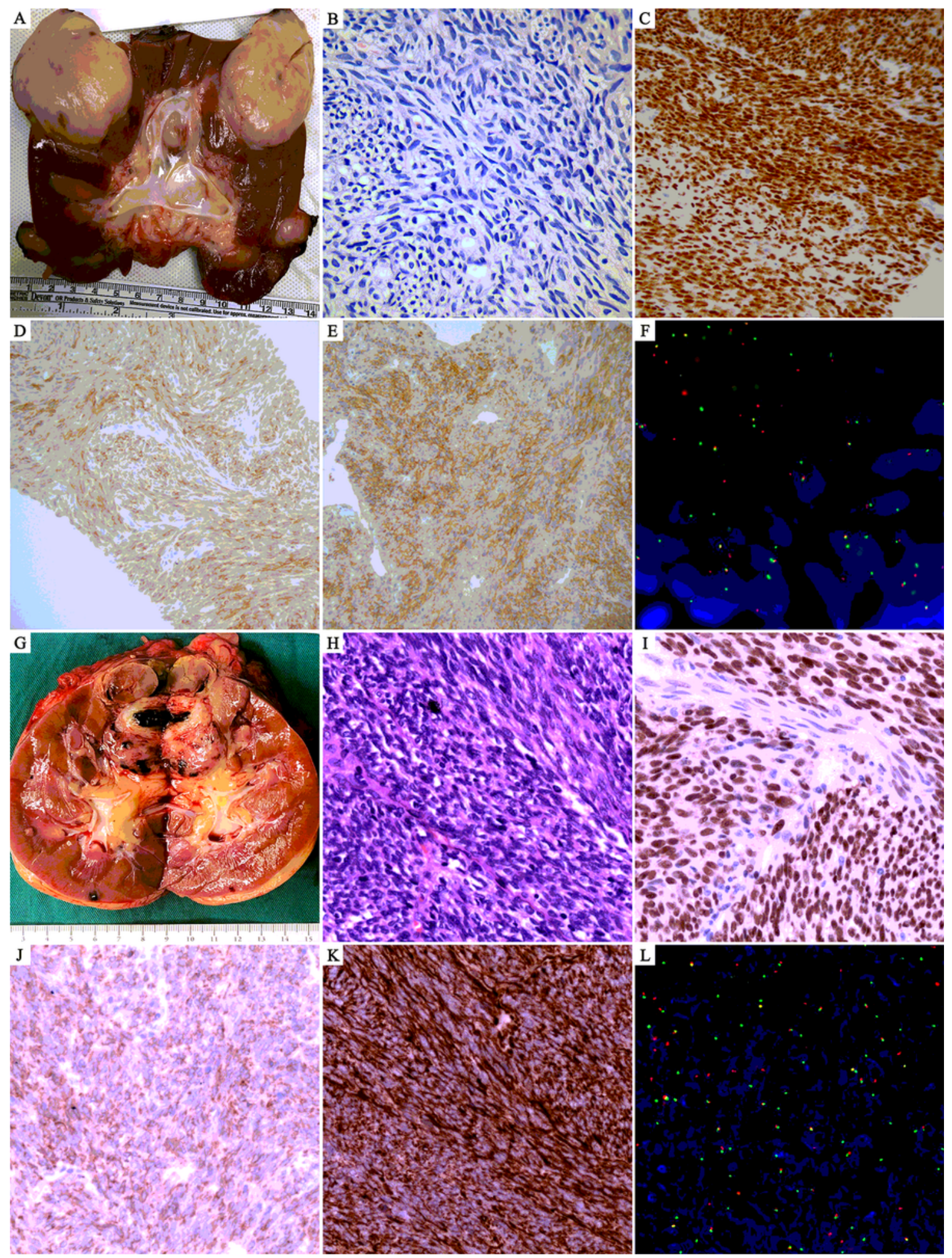

Figure 3

Histopathology of allograft synovial sarcoma Recipient 2: A: Specimen; B: HE-staining (200x); C: TLE1 (100x); D: Bcl-2 (100x); E: CD99 (200x); F: FISH test; Recipient 3: G: Specimen; H: HE-staining (200x); I: TLE1 (200x); J: Bcl-2 (200x); K: Vimentin (200x); L: FISH test. 

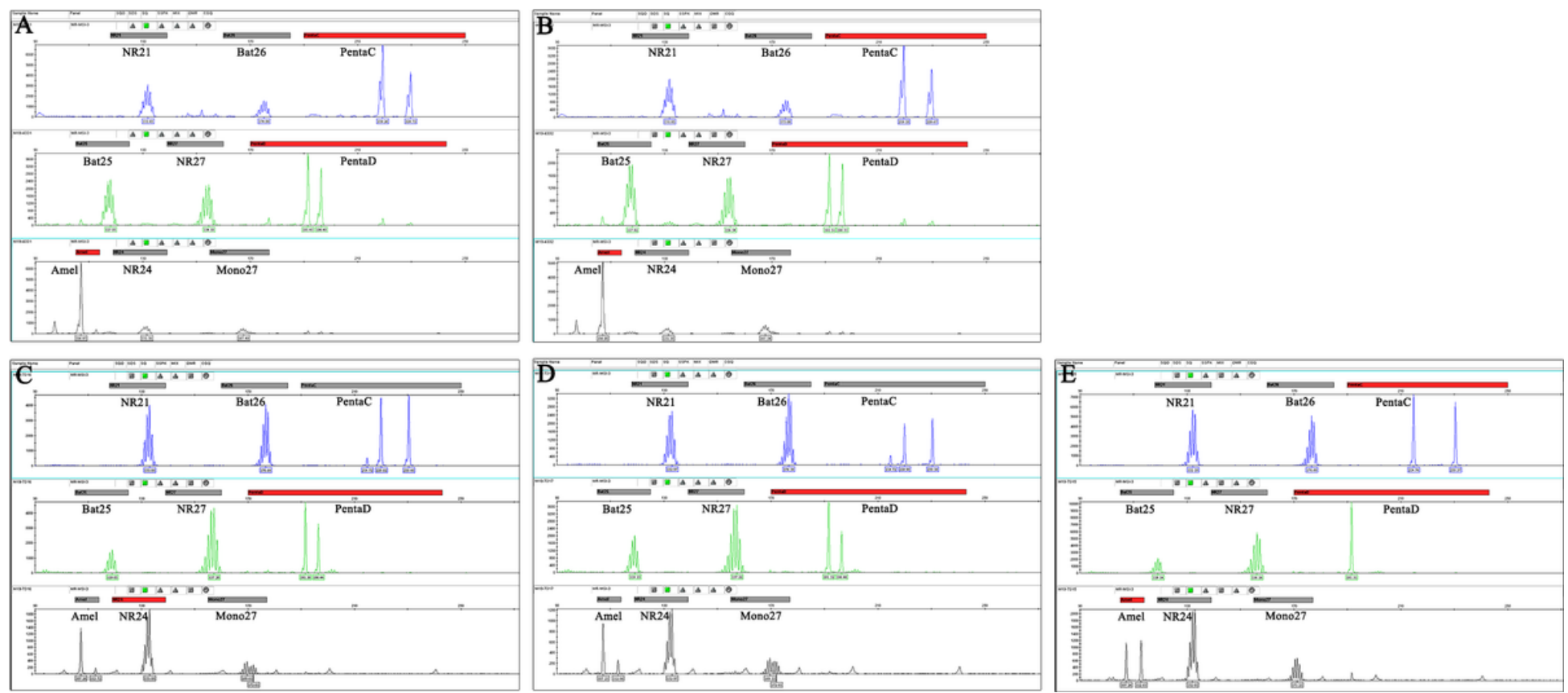

\section{Figure 4}

Length of detection locus by DNA microsatellite Recipient 2: A: Allograft tissue; B: Tumor tissue; Recipient 3: C: Allograft tissue; D: Tumor tissue; E: Blood of recipient.
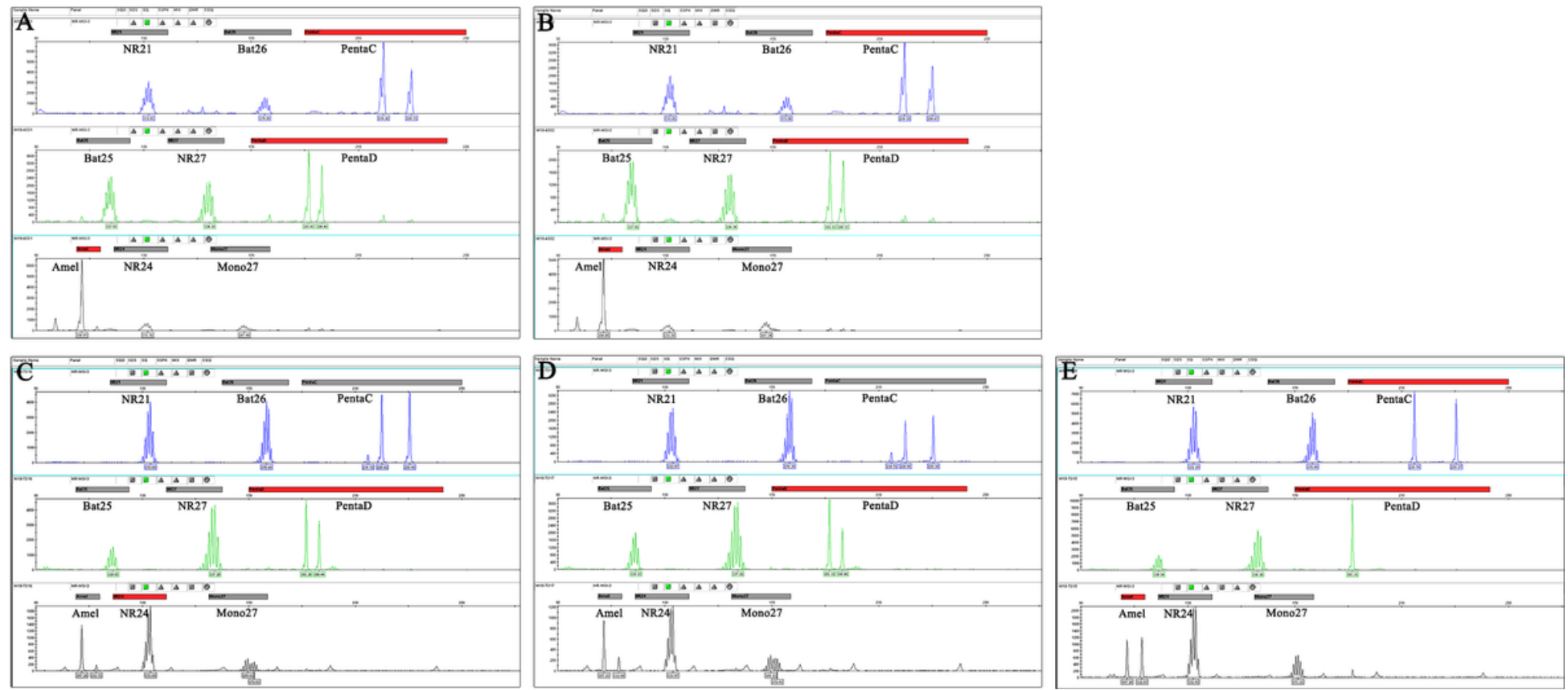

\section{Figure 4}

Length of detection locus by DNA microsatellite Recipient 2: A: Allograft tissue; B: Tumor tissue; Recipient 3: C: Allograft tissue; D: Tumor tissue; E: Blood of recipient.

\section{Supplementary Files}


This is a list of supplementary files associated with this preprint. Click to download.

- 1105182736040CAREchecklistEnglish20131.Jpeg

- 1105182736040CAREchecklistEnglish20131.Jpeg 